\title{
The Combination of STEM Tomography and STEM/EDS Analysis of NiSi Formation Related Defects in Semiconductor Wafer-foundries
}

\author{
B. Fu ${ }^{1}$, M. Gribelyuk ${ }^{1}$, L. Dumas ${ }^{1}$, C. Fang ${ }^{1}$, N. LaManque ${ }^{1}$, L. Hodgkins ${ }^{1}$, E. Chen ${ }^{1}$ \\ ${ }^{1 .}$ GLOBALFOUNDRIES, 400 Stone Break Rd Extension, Malta, NY, USA
}

Self-aligned nickel(platinum) silicidation process has been used in several semiconductor technology nodes to reduce source, drain, and gate resistance in metal-oxide-semiconductor (MOS) devices [1]. Formation of metal contacts to silicide regions is prone to defect formation. Residues on the Si surface prior to silicidation lead to blocked NiSi formation while residue left on top of the NiSi film causes poor metal contact to NiSi and increase of the contact resistance. Finally, unreacted Ni particles can cause electrical short if left on the wafer after silicidation. As semiconductor technology continues to scale down, these defects are generally smaller than the TEM sample thickness itself, which makes it difficult to obtain a complete understanding of the defect formation mechanism due to "projection effect" [2]. Therefore, electron tomography is generally required to understand the defect morphology and the defect location within the TEM lamella. In this study, a combination of STEM Tomography and STEM/EDS analysis of two defects in the $28 \mathrm{~nm}$ process technology are presented. It is shown, that using the combination of tomography and STEM/EDS analysis a complete understanding of the defect formation process and mechanism can be obtained.

Fig. 1 shows the results of a nickel silicide bridge defect from $\mathrm{PC}$ to $\mathrm{W}$ contact in a single bit failure of a SRAM cell. The cross-section STEM image (a) and EDS elemental image (b) confirm the bridge is NiSi. It also appears that the neighboring $\mathrm{W}$ contact lands on the NiSi at the S/D region (arrowed). However, from the 3D voltex view in STEM tomography, the W contact does not land on the NiSi (d). The isosurface view in Fig. 1e shows that the NiSi formation under this W contact is also blocked. This can be explained by the presence of silicon oxide particle located at the S/D region at the W contact, shown in the oxygen map in figure (c). This particle blocks the NiSi formation at S/D region and caused the shorter W contact. STEM tomography helped reveal the blocked NiSi defect in addition to the nickel silicide bridge defect found in TEM.

In Fig. 2a a particle is observed in the S/D region. Cross-section STEM and EDS analysis show, that the particle mainly contains $\mathrm{O}, \mathrm{N}$, and $\mathrm{Si}$ elements with some $\mathrm{Ni}$ signal detected in the particle core. However, NiSi at S/D region is still formed successfully below the particle (Figs. 2b,c). Since formation of NiSi requires clean $\mathrm{Si}$ surface it is difficult to understand how NiSi was formed. Figs.2d,e are two represented OrthoSlice views from 3D STEM tomography reconstruction volume, which clearly show that NiSi and the particle in Figs.2b,c are located in different planes within TEM sample, i.e. image in Fig. 2b suffers from the "projection effect". This explains why perfect NiSi film was formed. The particle is composed of some tiny NiSi present on top of the Si oxide particle, covered with nitride layer (Fig. 2e). Directly under the particle, no "NiSi" formation is observed as expected. The entire morphology of the particle and NiSi formation can be clearly seen in the 3D voltex image in Fig.2f.

The presented results illustrate unique capabilities of STEM tomography to understand the failure mechanisms in semiconductor devices and provide guidance for enhancement of device yield and performance.

References: 
[1] F. Deng et al, J. Appl. Phys. 81(1997), p. 8047.

[2] W. Zhao et al, Microsc. Microanal. 20 (2014), p. 362.
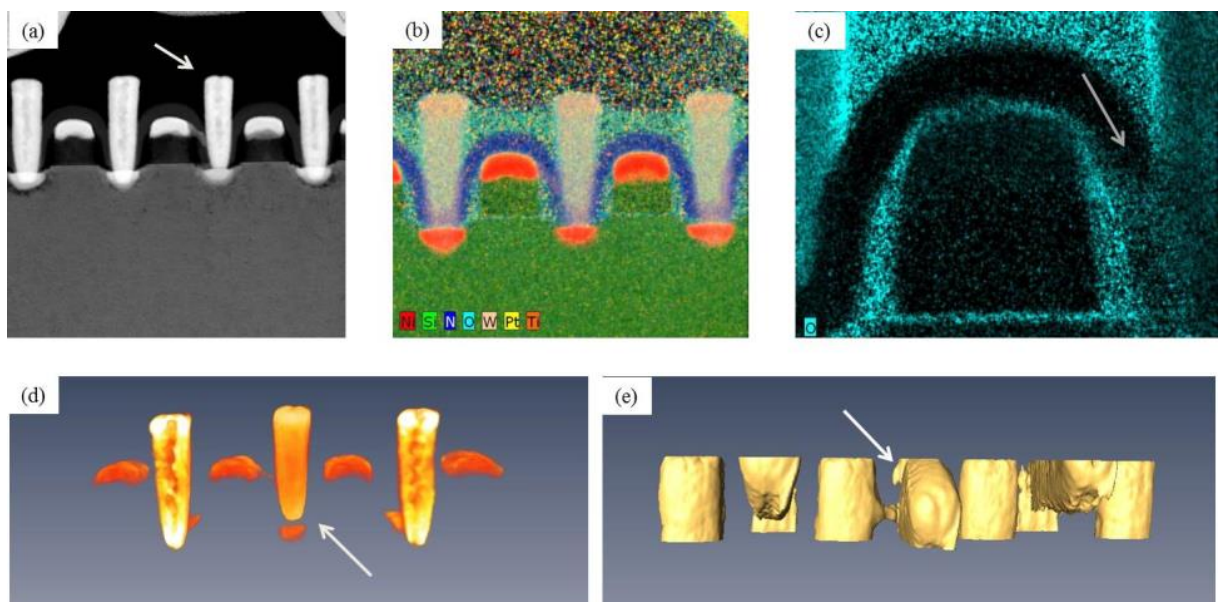

Figure 1. (a) STEM image showing S/D contact to gate short; (b) EDS elemental maps; (c) Enlarged view of oxygen map, noticing the extra oxygen signal (arrowed); (d) STEM tomography voltex visualization, showing contact is not landed on NiSi (arrowed); (e) STEM tomography isosurface visualization, showing part of the NiSi formation is blocked.
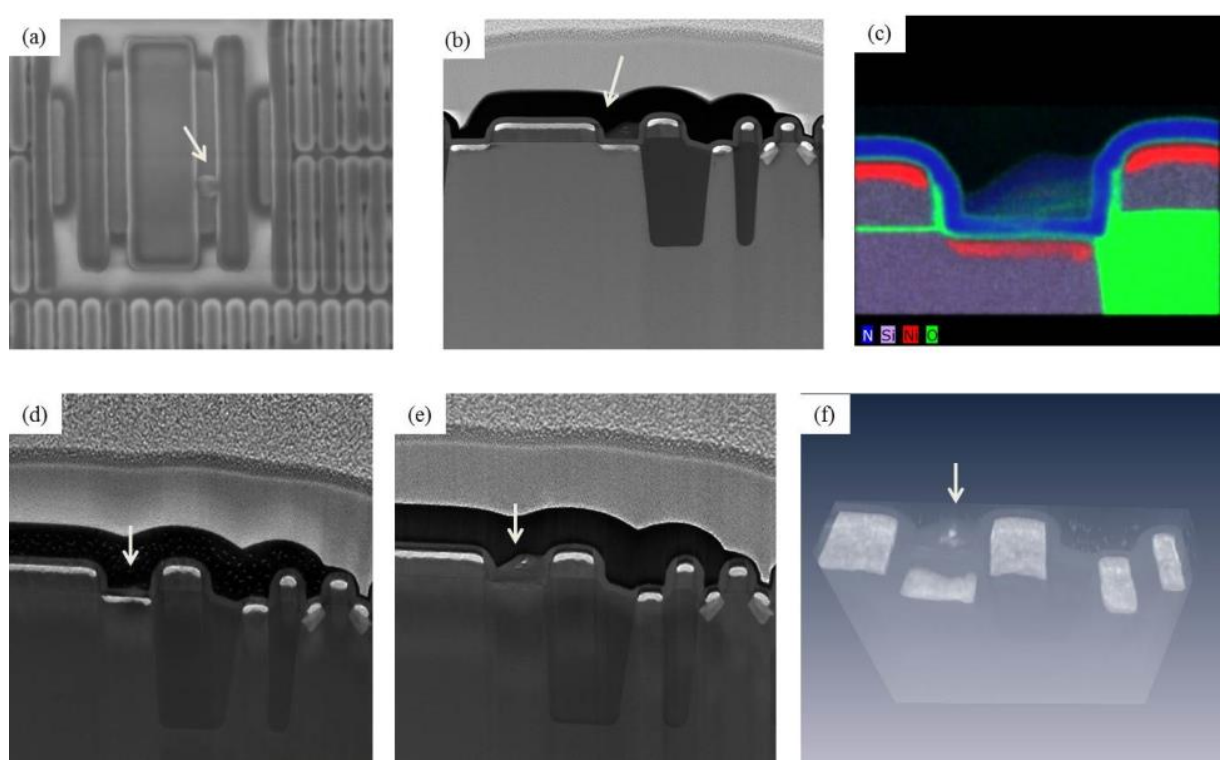

Figure 2. (a) Top-down SEM image of defect location (arrowed); (b) cross-section STEM image and (c) EDS elemental map of the defect location; (d) Reconstructed OrthoSlice before the particle; (e) Reconstructed OrthoSlice at the center of the particle; (f) STEM tomography voltex visualization of the particle. 